\section{Assistência à gestação e ao parto: resultados de dois estudos transversais em áreas pobres das regiões Norte e Nordeste do Brasil}

\author{
Health care during pregnancy and childbirth: \\ results of two cross-sectional surveys in poor areas \\ of North and Northeast Brazil
}

\author{
${ }^{1}$ Departamento de Medicina \\ Social, Universidade Federal \\ de Pelotas, Pelotas, Brasil. \\ 2 Departamento Materno- \\ Infantil, Universidade \\ Federal do Rio Grande, \\ Rio Grande, Brasil. \\ 3 Secretaria de Assistência \\ Social, Prefeitura Municipal \\ de Paulo Afonso, Paulo \\ Afonso, Brasil. \\ ${ }_{4}^{4}$ Coordenação Nacional, \\ Pastoral da Criança, \\ Curitiba, Brasil. \\ Correspondência \\ M. A. D. Chrestani \\ Residência de Medicina \\ Preventiva, Departamento \\ de Medicina Social, \\ Universidade Federal de \\ Pelotas. \\ Av. Duque de Caxias 250, \\ 3o piso, Pelotas, RS \\ 96030-002, Brasil. \\ machrestani@uol.com.br
}

\begin{abstract}
In 2002 and 2005, two surveys were conducted in poor areas of North and Northeast Brazil to evaluate prenatal care received by mothers of children under five years old. The study covered 1,528 and 1,529 children in 2002 and 2005, respectively. In this three-year period, maternal schooling and monthly family income increased by 1.5 years and US\$50.00, respectively, availability of safe drinking water increased from $23 \%$ to $41 \%$, the proportion of pregnant woman with six or more prenatal visits increased from $42 \%$ to $52 \%$, and immunization against neonatal tetanus increased from $54 \%$ to $77 \%$. Coverage of uterine height evaluation increased from $54 \%$ to $77 \%$. Testing for HIV and syphilis virtually doubled, from 8\% to 16\%. Meanwhile, gynecological examinations dropped from $41 \%$ to $31 \%$ and counseling for breastfeeding decreased from 66\% to 55\%. Despite improvements, the study's results show that the target indicators in these areas fall short of those in more developed regions of Brazil. Efforts should be made to reduce this gap.
\end{abstract}

Delivery of Health Care; Pregnancy; Parturition; Maternal and Child Health

\author{
Maria Aurora D. Chrestani 1 \\ Iná S. Santos 1 \\ Juraci A. Cesar 2 \\ Leonardo S. Winckler 2 \\ Tatiane S. Gonçalves 3 \\ Nelson A. Neumann 4
}

\section{Introdução}

A assistência adequada à gestação e ao parto é essencial para reduzir os índices de morbimortalidade materno-infantil. Estima-se que um quarto dos óbitos infantis e a quase totalidade dos óbitos maternos decorram da prestação de cuidados inadequados desde o início da gestação até o pós-parto imediato 1 .

O Brasil possui índices de morbimortalidade materno-infantil bastante elevados, se comparados aos países desenvolvidos 2 . Em 2003, o coeficiente de mortalidade materna foi de 51,7 óbitos por 100 mil nascidos vivos e o de mortalidade infantil, de 24,4 por mil 3,4. Estes índices são cerca de seis vezes maiores em relação a países desenvolvidos 2 .

Preocupado com estes índices, o governo federal criou no ano 2000 o Programa de Humanização do Pré-Natal (PHPN) 5. O objetivo deste programa é melhorar a cobertura, reduzir os índices de morbimortalidade materno-infantil e estabelecer diretrizes da atenção pré-natal e da assistência ao parto no Sistema Único de Saúde (SUS). Até 2004, cerca de $80 \%$ dos municípios brasileiros já estavam sendo beneficiados pelo PHPN e dados do Ministério da Saúde mostram que a cobertura da assistência à gestação e ao parto vem aumentando em todas as regiões do país nos últimos anos 5. Porém, pouco se sabe sobre a qualidade dos serviços oferecidos, especialmente nas regiões Norte e Nordeste, as mais 
pobres do país e as de mais difícil acesso ${ }^{1}$. Por exemplo, em 2003 , somente $30 \%$ das gestantes destas regiões realizaram seis ou mais consultas de pré-natal contra $60 \%$ na Região Sul ${ }^{4}$. Além de cobertura claramente insuficiente, evidencia-se novamente que novos programas e tecnologias alcançam primeiramente aqueles em melhor condição, para somente mais tarde atingir os mais necessitados 6 .

Em 2002, por solicitação da Pastoral da Criança e do Fundo das Nações Unidas para a Infância (UNICEF), realizou-se estudo transversal visando avaliar indicadores básicos de saúde materno-infantil em 18 áreas de 9 dos 36 municípios mais pobres destas regiões. Em 2005, decidiu-se retornar às mesmas áreas destes municípios e reavaliar os mesmos indicadores. Este artigo apresenta os resultados obtidos nestas duas ocasiões em relação à assistência à gestação e ao parto e discute os seus diferenciais.

\section{Metodologia}

Os dois estudos apresentados neste artigo foram realizados nos anos de 2002 e 2005, em 18 áreas de 9 dos 36 municípios mais pobres das regiões Norte e Nordeste do Brasil. Todos estes municípios foram classificados com base em dados do UNICEF e da Pastoral da Criança e apenas nove deles indicados para serem incluídos neste estudo. Dois destes municípios pertencem à Região Norte (Garrafão do Norte e Santa Cruz do Arari, Estado do Pará) e os demais, à Região Nordeste (Caracol, Estado do Piauí; Macururé, Estado da Bahia; Graça e Arneiroz, no Estado do Ceará; Natuba e Mari, Estado da Paraíba; e São Pedro, Estado do Rio Grande do Norte).

A idéia destas organizações era avaliar a saúde infantil nestes municípios a partir do monitoramento de alguns indicadores básicos de saúde, dentre os quais aqueles relacionados à assistência à gestação e ao parto entre crianças menores de cinco anos. Tanto em 2002 quanto em 2005, foram escolhidas duas áreas pobres em cada um destes municípios conforme descrito mais adiante nesta seção. O delineamento utilizado foi o transversal por ser o mais adequado à avaliação simultânea de diversas exposições e desfechos 7 .

Eram elegíveis para o estudo os locais de atuação da Pastoral da Criança, uma vez que esta, invariavelmente, alcança as famílias de mais baixo nível sócio-econômico dentro de cada município 8,9.

Para o cálculo do tamanho de amostra dos dois estudos considerou-se erro alfa de 0,05 , erro beta de 0,20 , precisão ou erro máximo de 3,6 pontos percentuais, efeito de delineamento de 1,8 , prevalência de desfechos variando de $20 \%$ a $80 \%$. Isso resultou em um tamanho amostral mínimo de 1.328 crianças. A esse valor foram acrescidos $10 \%$ (133 crianças) para eventuais perdas. Assim, estes estudos deveriam incluir pelo menos 1.461 crianças menores de cinco anos.

Decidiu-se $a$ priori que duas áreas seriam visitadas por município. Caso fosse encontrada apenas uma área de atuação da Pastoral da Criança no município, uma outra com as mesmas características (em termos de tipo de construção da moradia, calçamento de rua, disponibilidade de energia elétrica, água tratada, rede de esgotos, escolas e serviços de saúde), era escolhida. Considerando que o estudo deveria cobrir cerca de 1.500 crianças em nove municípios, seria necessário incluir 167 crianças por município. A fim de recrutar número igual de criança por área, arredondou-se para 168. Assim, em cada área seriam selecionadas 84 crianças. Após definição das áreas a serem incluídas no estudo, estas foram mapeadas e as que pertenciam à zona urbana tinham suas quadras e esquinas numeradas e sorteadas de forma aleatória. Na zona rural, as áreas eram delimitadas por rios, montanhas, estradas e pontes, cada uma delas numeradas e, também, escolhidas de forma aleatória. A partir do ponto inicial, foram visitados todos os domicílios de forma consecutiva até completar as 84 crianças por área.

As informações foram coletadas através da aplicação, em nível domiciliar, de dois questionários padronizados e previamente testados à mãe ou pessoa responsável pela guarda da criança. Um dos questionários buscava informações sobre a família quanto a características demográficas, grau de escolaridade, nível sócio-econômico dos pais, condições de habitação e saneamento e presença de equipamentos domésticos por domicílio. Estas informações foram coletadas através das seguintes variáveis: cor da pele (definida pelo entrevistador em branca, parda/morena e preta) e idade da mãe em anos completos, presença de companheiro, grau de escolaridade da mãe, renda familiar mensal de todos os moradores do domicílio no mês imediatamente anterior à entrevista, número de moradores por domicílio, tipo de construção da moradia, disponibilidade de água encanada dentro de casa ou no pátio/terreno, origem da água usada para beber (rede pública, rio/lagoa/açude, cisterna/poço), tipo de sanitário (com descarga, sem descarga, casinha/fossa negra), energia elétrica (regular ou clandestina) e eletrodomésticos em condições de funcionamento.

No outro questionário, investigou-se a assistência recebida durante a gestação e o parto e algumas características das crianças. Interrogou- 
se quanto à realização de pré-natal, mês de início e número de consultas de pré-natal, imunização completa contra tétano (número de doses recebidas e quando isto ocorreu) e imunização prévia. Investigou-se ainda se, durante o pré-natal, houve verificação da pressão arterial, pesagem da gestante, avaliação dos batimentos cárdiofetais (utilizando sonar Doppler), altura uterina e presença de edema de membros inferiores. A realização de exame citopatológico de colo uterino com coleta de material (Papanicolaou) e de mamas, recebimento de orientação sobre a amamentação e prescrição de sulfato ferroso durante a gestação, realização de exame de urina, fezes, sangue, anti-HIV, sífilis e ultra-som obstétrico foram também explorados. Além disso, perguntou-se sobre local de realização, tipo de parto e de profissional que o realizou, peso de nascimento da criança em gramas e se havia sido feita a revisão puerperal nos trinta dias seguintes ao nascimento do bebê. Se no domicílio existisse mais de uma criança menor de cinco anos, aplicava-se questionário buscando avaliar o pré-natal de todas elas.

Tanto em 2002 quanto em 2005, foram selecionados e treinados seis entrevistadores graduados em Ciências Sociais pela Universidade Federal de Sergipe. A coordenadora do trabalho de campo (T.S.G.) foi a mesma nos dois estudos. O treinamento consistiu de leitura do manual de instruções e simulações de entrevista. Após, foi realizado o estudo piloto na cidade de Aracaju, Estado de Sergipe, local de residência da maioria dos entrevistadores.

Os dados coletados foram duplamente digitados, comparados, corrigidos e sua consistência avaliada através do programa Epi Info 6.04 (Centers for Disease Control and Prevention, Atlanta, Estados Unidos). A magnitude das associações foi avaliada através do teste de qui-quadrado de Pearson. Todas as análises foram realizadas utilizando-se o programa Stata versão 7.0 (Stata Corp., College Station, Estados Unidos).

O controle de qualidade nos dois estudos foi realizado a partir da revisão dos questionários e repetição parcial de 5\% das entrevistas. Este estudo foi submetido e aprovado pelo Comitê de Ética em Pesquisas na Área da Saúde da Fundação Universidade Federal de Rio Grande, Rio Grande do Sul, Brasil.

\section{Resultados}

Em 2002 foram identificadas 1.554 crianças menores de cinco anos, sendo obtidas informações sobre 1.528 delas, o que representa índice de perdas de 1,7\%. Em 2005, visitando as mesmas áreas, foram encontradas 1.589 crianças de mesma faixa etária. Deste total não foi possível obter informações sobre 55 delas, totalizando 3,6\% de perdas. Foram incluídos no estudo, em média, 170 crianças por município. Esta diferença se deve ao fato de um domicilio situar-se muito distante do outro impossibilitando, sobretudo nas áreas rurais, que um entrevistador verificasse com os demais os números de entrevistas já realizadas. Ainda, se o número de entrevistas desejadas já houvesse sido realizado, mas, no domicílio, residissem mais de uma criança menor de cinco anos, aplicava-se questionário buscando avaliar o pré-natal realizado na gravidez de todas elas. Por esta razão, o número de entrevistas realizadas foi um pouco superior ao inicialmente estimado.

A Tabela 1 descreve as características demográficas e a escolaridade das mães em 2002 e em 2005. Houve significativa redução no percentual de mulheres de cor da pele branca e aumento na proporção daquelas de cor parda/mulata e preta. A média de idade das mães foi pouco superior a 28 anos nos dois estudos. Observou-se redução da ordem de $30 \%$ na taxa de analfabetismo e o percentual de mães com nove anos ou mais de escolaridade triplicou, passando de $9 \%$ para $28 \%$ no período.

A Tabela 2 mostra que houve aumento na renda média familiar e no percentual de famílias recebendo dois ou mais salários mínimos mensais. Entre 2002 e 2005, a renda média aumentou em $55 \%$ e a mediana em $81 \%$. As condições de habitação e saneamento também melhoraram. A proporção de domicílios com utilização de água proveniente de rede pública passou de $23 \%$ para $41 \%$; e a de domicílios sem nenhum tipo de sanitário caiu de $40 \%$ para $30 \%$ no período. O acesso à energia elétrica, com ligação de forma regular à rede, aumentou de $72 \%$ para $82 \%$ e a disponibilidade de eletrodomésticos também melhorou, sobretudo a posse de geladeira, que passou de 25\% em 2002 para 38\% em 2005.

A Tabela 3 mostra que houve, no geral, aumento na quantidade e melhora na qualidade dos cuidados oferecidos à gestante durante o pré-natal destas crianças. Praticamente nove em cada dez gestantes iniciaram o pré-natal em 2005. No entanto, não houve diferença significativa em relação ao trimestre de início do pré-natal entre os dois estudos. A proporção de mulheres que realizou seis ou mais consultas aumentou de $42 \%$ para $52 \%$. Em termos de média, isto significou aumento de aproximadamente uma consulta por gestação no período. Por fim, a taxa de imunização antitetânica passou de 54\% em 2002 para $77 \%$ em 2005.

Ainda na Tabela 3 é possível verificar que, em 2005, a quase totalidade das mães teve veri- 
Características demográficas e escolaridade das mães de menores de cinco anos residentes em municípios pobres nas regiões Norte e Nordeste do Brasil em 2002 e 2005.

\begin{tabular}{|c|c|c|c|}
\hline \multirow[t]{2}{*}{ Variável } & \multicolumn{2}{|c|}{ Total } & \multirow[t]{2}{*}{ Valor de $\mathrm{p}$} \\
\hline & 2002 & 2005 & \\
\hline Cor da pele & & & $<0,001$ \\
\hline Branca & $27,5 \%$ & $15,3 \%$ & \\
\hline Parda/Mulata & $63,7 \%$ & $73,4 \%$ & \\
\hline Preta & $7,8 \%$ & $11,3 \%$ & \\
\hline \multicolumn{4}{|l|}{ Idade (anos completos) } \\
\hline$<20$ & $9,7 \%$ & $9,2 \%$ & 0,122 \\
\hline $20-29$ & $54,1 \%$ & $58,2 \%$ & \\
\hline $30-39$ & $23,7 \%$ & $21,8 \%$ & \\
\hline 40 ou mais & $12,5 \%$ & $10,7 \%$ & \\
\hline Média idade (desvio-padrão) & $28,6(9,2)$ & $28,2(9,1)$ & \\
\hline \multicolumn{4}{|l|}{ Situação conjugal } \\
\hline Tem marido/companheiro & $84,8 \%$ & $86,9 \%$ & 0,095 \\
\hline Vive junto com o marido/companheiro & $81,7 \%$ & $94,1 \%$ & $<0,001$ \\
\hline \multicolumn{4}{|l|}{ Escolaridade da mãe (anos completos) } \\
\hline Não alfabetizadas & $37,2 \%$ & $28,8 \%$ & $<0,001$ \\
\hline Nenhum & $21,1 \%$ & $15,8 \%$ & $<0,001$ \\
\hline $1-3$ & $35,6 \%$ & $19,0 \%$ & \\
\hline $4-8$ & $34,4 \%$ & $37,5 \%$ & \\
\hline 9 ou mais & $9,0 \%$ & $27,6 \%$ & \\
\hline Média escolaridade (desvio-padrão) & $3,5(3,2)$ & $5,1(3,6)$ & \\
\hline Total (n) & $100,0 \%(1.528)$ & $100,0 \%(1.529)$ & \\
\hline
\end{tabular}

ficados pelo menos uma vez a pressão arterial, peso e ausculta dos batimentos cárdio-fetais. $\mathrm{O}$ aumento mais expressivo foi observado em relação à mensuração da altura uterina, que passou de $54 \%$ em 2002 para $77 \%$ em 2005 . No entanto, houve redução significativa na realização de exame ginecológico, das mamas e na orientação para a amamentação no período estudado. Apesar disso, houve aumento significativo na realização de exame citopatológico do colo uterino. O rastreamento para HIV e sífilis também aumentou neste período. A proporção de gestantes que fez exame para detecção de HIV e sífilis duplicou, passando de 7\% em 2002 para cerca de $15 \%$ em 2005. A realização de ultra-som obstétrico também aumentou, passando de $44 \%$ em 2002 para 64\% em 2005 (Tabela 3).

Finalmente, a Tabela 4 mostra pequenas diferenças entre os dois estudos quanto à assistência ao parto, peso ao nascer e realização de revisão no puerpério. Embora a grande maioria dos nascimentos tenha ocorrido em hospital ou maternidade, $20 \%$ das crianças nasceram no próprio domicílio, casa de parto ou em ambulância. Tanto em 2002 quanto em 2005, cerca de um terço dos partos não foi realizado por médico ou enfermeira. Mesmo que a grande maioria dos partos tenha sido assistida por algum profissional da saúde, chama a atenção o fato de, nos dois inquéritos, $3 \%$ terem ocorrido sem qualquer tipo de assistência. Houve um ligeiro aumento, porém significativo, na freqüência de cesarianas, que passou de $13 \%$ em 2002 para $16 \%$ em 2005 . O registro do peso ao nascer, confirmado através da carteira da gestante, melhorou, passando de $74 \%$ em 2002 para $81 \%$ em 2005. O mesmo não ocorreu com a revisão no puerpério. Somente $18 \%$ das mães nos dois estudos retornaram à consulta médica nas quatro semanas seguintes ao parto para fazer revisão puerperal.

\section{Discussão}

Este estudo mostra que, entre 2002 e 2005, houve substancial melhora no nível sócio-econômico e nas condições de moradia das famílias de menores de cinco anos nas áreas estudadas. Mostra também que, neste período, houve im- 
Renda familiar e condições de moradia entre menores de cinco anos residentes em municípios pobres nas regiões Norte e Nordeste do Brasil em 2002 e 2005.

\begin{tabular}{|c|c|c|c|}
\hline \multirow[t]{2}{*}{ Variável } & \multicolumn{2}{|c|}{ Total } & \multirow[t]{2}{*}{ Valor de $\mathrm{p}$} \\
\hline & 2002 & 2005 & \\
\hline \multicolumn{4}{|l|}{ Renda familiar mensal (salários mínimos) } \\
\hline$<1$ & $64,9 \%$ & $60,8 \%$ & $<0,001$ \\
\hline $1-1,9$ & $24,1 \%$ & $23,2 \%$ & \\
\hline 2 ou mais & $11,0 \%$ & $16,0 \%$ & \\
\hline Média (desvio-padrão) em R\$ & $176,00(188,00)$ & $273,00(307,00)$ & \\
\hline Mediana em R\$ & 110,00 & 200,00 & \\
\hline \multicolumn{4}{|l|}{ Número de moradores por domicílio } \\
\hline 3 & $11,0 \%$ & $15,8 \%$ & $<0,001$ \\
\hline 4 ou 5 & $34,1 \%$ & $39,7 \%$ & \\
\hline 6 ou 7 & $26,4 \%$ & $24,7 \%$ & \\
\hline 8 ou mais & $28,4 \%$ & $19,8 \%$ & \\
\hline Média moradores (desvio-padrão) & $6,4(2,7)$ & $5,7(2,4)$ & \\
\hline \multicolumn{4}{|l|}{ Tipo de construção da moradia } \\
\hline Tijolo/Bloco & $62,1 \%$ & $73,0 \%$ & $<0,001$ \\
\hline Taipa & $22,8 \%$ & $12,6 \%$ & \\
\hline Madeira & $15,1 \%$ & $14,4 \%$ & \\
\hline \multicolumn{4}{|l|}{ Água encanada } \\
\hline Dentro de casa & $28,3 \%$ & $31,2 \%$ & $<0,001$ \\
\hline No pátio/terreno & $18,0 \%$ & $21,9 \%$ & \\
\hline Não tem & $53,7 \%$ & $46,9 \%$ & \\
\hline \multicolumn{4}{|l|}{ Origem da água usada para beber } \\
\hline Rede pública & $23,5 \%$ & $41,2 \%$ & $<0,001$ \\
\hline Cisterna/Poço & $45,1 \%$ & $33,4 \%$ & \\
\hline Rio/Lago/Lagoa/Açude & $19,4 \%$ & $11,0 \%$ & \\
\hline Outros & $12,0 \%$ & $14,4 \%$ & \\
\hline \multicolumn{4}{|l|}{ Tipo de privada } \\
\hline Sanitário com descarga & $16,0 \%$ & $21,2 \%$ & $<0,001$ \\
\hline Sanitário sem descarga & $31,5 \%$ & $29,8 \%$ & \\
\hline Casinha/Fossa negra & $12,9 \%$ & $18,6 \%$ & \\
\hline Não tem & $39,6 \%$ & $30,4 \%$ & \\
\hline \multicolumn{4}{|l|}{ Domicílio com energia elétrica } \\
\hline Sim, direto & $71,6 \%$ & $82,2 \%$ & $<0,001$ \\
\hline Sim, clandestina & $4,2 \%$ & $5,6 \%$ & \\
\hline Não & $24,2 \%$ & $12,2 \%$ & \\
\hline \multicolumn{4}{|l|}{ Disponibilidade de eletrodomésticos } \\
\hline Rádio & $64,5 \%$ & $65,3 \%$ & 0,640 \\
\hline Televisão & $56,3 \%$ & $66,1 \%$ & $<0,001$ \\
\hline Geladeira & $24,9 \%$ & $37,6 \%$ & $<0,001$ \\
\hline Filtro de água & $28,1 \%$ & $28,2 \%$ & 0,945 \\
\hline Fogão a gás & $73,9 \%$ & $72,3 \%$ & 0,574 \\
\hline Fogão a lenha/carvão & $72,6 \%$ & $62,5 \%$ & $<0,001$ \\
\hline Total $(n)$ & $100,0 \%(1.528)$ & $100,0 \%(1.529)$ & \\
\hline
\end{tabular}

portantes avanços na assistência à gestação e ao parto, embora permaneçam deficiências se comparados aos observados em regiões mais desenvolvidas do país.
Ao interpretar estes resultados há que considerar pelo menos duas limitações que podem ter afetado este estudo. Primeiro, o erro de recordatório: todos os resultados apresentados 
Consultas de pré-natal, imunização antitetânica e exames clínicos e laboratoriais realizados por mães de menores de cinco anos residentes em municípios pobres nas regiões Norte e Nordeste do Brasil em 2002 e 2005.

\begin{tabular}{|c|c|c|c|}
\hline \multirow[t]{2}{*}{ Variável } & \multicolumn{2}{|c|}{ Total } & \multirow[t]{2}{*}{ Valor de $p$} \\
\hline & 2002 & 2005 & \\
\hline Fez pré-natal & $86,0 \%$ & $89,8 \%$ & 0,001 \\
\hline \multicolumn{4}{|l|}{ Trimestre em que iniciaram o pré-natal } \\
\hline Primeiro & $67,8 \%$ & $70,3 \%$ & 0,383 \\
\hline Segundo & $29,1 \%$ & $26,7 \%$ & \\
\hline Terceiro & $3,2 \%$ & $3,0 \%$ & \\
\hline \multicolumn{4}{|l|}{ Número de consultas realizadas } \\
\hline 0 & $17,2 \%$ & $8,8 \%$ & $<0,001$ \\
\hline $1-5$ & $41,0 \%$ & $39,4 \%$ & \\
\hline 6 ou mais & $41,8 \%$ & $51,8 \%$ & \\
\hline Média de consultas (desvio padrão) & $4,7(3,4)$ & $5,5(3,8)$ & \\
\hline Imunização contra tétano neonatal & $54,3 \%$ & $77,3 \%$ & $<0,001$ \\
\hline \multicolumn{4}{|l|}{ Verificação da(o) } \\
\hline Pressão arterial & $92,4 \%$ & $97,2 \%$ & $<0,001$ \\
\hline Peso da gestante & $92,8 \%$ & $97,7 \%$ & $<0,001$ \\
\hline Batimentos cardíacos do feto & $85,3 \%$ & $91,7 \%$ & $<0,001$ \\
\hline Altura uterina & $54,4 \%$ & $77,1 \%$ & $<0,001$ \\
\hline Edema de membros inferiores & $64,7 \%$ & $68,0 \%$ & 0,080 \\
\hline \multicolumn{4}{|l|}{$\mathrm{Se} \ldots$} \\
\hline Fez exame ginecológico & $41,4 \%$ & $30,8 \%$ & $<0,001$ \\
\hline Teve o seio examinado & $42,5 \%$ & $38,3 \%$ & 0,031 \\
\hline Recebeu orientação sobre amamentação & $66,3 \%$ & $55,4 \%$ & $<0,001$ \\
\hline Recebeu sulfato ferroso durante a gestação & $81,3 \%$ & $84,9 \%$ & 0,013 \\
\hline \multicolumn{4}{|l|}{ Tipo de exame } \\
\hline Urina & $77,3 \%$ & $81,6 \%$ & 0,007 \\
\hline Fezes & $56,3 \%$ & $57,0 \%$ & 0,738 \\
\hline Sangue & $77,0 \%$ & $84,2 \%$ & $<0,001$ \\
\hline Citopatológico de colo uterino & $12,3 \%$ & $15,5 \%$ & 0,019 \\
\hline Anti-HIV & $7,6 \%$ & $17,7 \%$ & $<0,001$ \\
\hline Detecção de sífilis & $7,2 \%$ & $13,9 \%$ & $<0,001$ \\
\hline Ultra-som obstétrico & $43,8 \%$ & $64,0 \%$ & $<0,001$ \\
\hline Total (n) & $100,0 \%(1.528)$ & $100,0 \%(1.529)$ & \\
\hline
\end{tabular}

neste artigo foram coletados durante uma única abordagem com base apenas no relato das mães, exceto para o peso ao nascer, que foi confirmado na carteira da gestante ou no cartão de saúde da criança. $\mathrm{O}$ viés de recordatório, se presente, provavelmente teve menor efeito entre as mães que tiveram suas gestações mais recentemente. Segundo, a falta de representatividade: este estudo incluiu apenas algumas áreas dos municípios mais pobres, logo seus resultados não os representam como um todo.

O erro de recordatório, que pode ter subestimado os resultados aqui apresentados, ocorreu tanto em 2002 quanto em 2005 porque a metodologia utilizada foi a mesma. A falta de representa- tividade compromete a validade externa, mas a generalização dos achados não era um objetivo deste estudo. A idéia principal era mostrar a evolução de alguns indicadores de saúde maternoinfantil ao longo de três anos em algumas áreas destes municípios. Isto não impede, no entanto, que estes dados sirvam de base para estimativas em áreas geográficas e populações similares às aqui estudadas.

Há diversos estudos mostrando melhora no poder aquisitivo das famílias no Brasil, sobretudo das mais pobres 10 . Isto decorre, principalmente, da expansão da rede de programas sociais do governo federal, com destaque para o Programa Bolsa-Família. Este programa, implementado em 
Assistência ao parto, peso ao nascer e revisão de puerpério para menores de cinco anos residentes em municípios pobres nas regiões Norte e Nordeste do Brasil em 2002 e 2005.

\begin{tabular}{|c|c|c|c|}
\hline \multirow[t]{2}{*}{ Variável } & \multicolumn{2}{|c|}{ Total } & \multirow[t]{2}{*}{ Valor de $p$} \\
\hline & 2002 & 2005 & \\
\hline \multicolumn{4}{|l|}{ Local de nascimento } \\
\hline Hospital/Maternidade & $80,4 \%$ & $80,6 \%$ & $<0,001$ \\
\hline No próprio domicílio & $15,2 \%$ & $11,2 \%$ & \\
\hline Casa de parto/Ambulância & $4,4 \%$ & $8,2 \%$ & \\
\hline \multicolumn{4}{|l|}{ Quem fez o parto } \\
\hline Médico & $41,1 \%$ & $41,9 \%$ & 0,038 \\
\hline Enfermeira & $26,0 \%$ & $22,6 \%$ & \\
\hline Parteira & $29,4 \%$ & $32,8 \%$ & \\
\hline Ninguém & $3,5 \%$ & $2,6 \%$ & \\
\hline \multicolumn{4}{|l|}{ Tipo de parto } \\
\hline Normal & $86,8 \%$ & $83,5 \%$ & 0,049 \\
\hline Cesariana & $13,2 \%$ & $15,6 \%$ & \\
\hline \multicolumn{4}{|l|}{ Peso ao nascer (gramas) } \\
\hline$<2.500$ & $8,2 \%$ & $5,8 \%$ & 0,035 \\
\hline $2.500-2.990$ & $15,1 \%$ & $17,7 \%$ & \\
\hline $3.000-3.499$ & $39,2 \%$ & $38,7 \%$ & \\
\hline 3.500 ou mais & $37,5 \%$ & $37,7 \%$ & \\
\hline Peso ao nascer confirmado & $73,9 \%$ & $81,4 \%$ & $<0,001$ \\
\hline Fizeram revisão puerperal & $18,5 \%$ & $18,6 \%$ & 0,949 \\
\hline Total (n) & $100,0 \%(1.528)$ & $100,0 \%(1.529)$ & \\
\hline
\end{tabular}

2004, tem alcançado aqueles com maior necessidade como a população incluída neste estudo. Entre as famílias estudadas, $40 \%$ eram beneficiadas pelo Bolsa-Família. O aumento médio de renda mensal no período foi de aproximadamente $\mathrm{R} \$ 100,00$, o que possibilitou investimentos que levaram à melhoria nas condições de habitação, aqui evidenciada pelo tipo de construção da moradia, disponibilidade de água encanada dentro de casa e de sanitário com descarga. Além disso, a posse de alguns eletrodomésticos, sobretudo geladeira, melhorou de forma significativa. Isto é particularmente importante, por permitir o consumo de alimentos armazenados em condições adequadas, o que contribui para a redução de algumas doenças, particularmente as gastrintestinais de origem infecciosa. Além disso, evita o desperdício, o que reduz os gastos da família com alimentação.

As diferenças encontradas entre a cor da pele das mães nos dois estudos pode ser decorrente de erro de classificação, pois esta variável foi coletada através da observação do próprio entrevistador e, dos cinco entrevistadores participantes do estudo em 2005, somente um havia participado do de 2002. O nível de escolaridade no Brasil tem melhorado de forma expressiva, sobretudo nos últimos anos 2. No entanto, há que considerar neste estudo um possível efeito de coorte. Cerca de $60 \%$ das crianças estudadas em 2002 não participaram do estudo de 2005 porque já tinham alcançado cinco anos de idade não sendo, portanto, elegíveis para este estudo. Como a taxa de fecundidade no Brasil tem caído, mas não entre as mais jovens, é possível que as mães de maior idade tenham sido substituídas por outras mais jovens com maior grau de escolaridade. Logo, a mudança observada decorre não somente de as mães terem estudado mais ao longo destes três anos, mas, sobretudo, por terem sido substituídas por outras, mais jovens e com maior grau de escolaridade. Daí a explicação para o fato de, em tão curto espaço de tempo, a escolaridade materna ter aumentado, em média, 1,5 anos.

A assistência à gestação e ao parto tem melhorado no Brasil como um todo 1 . Na cidade de Pelotas, Estado do Rio Grande do Sul, por exemplo, estudos de coorte realizados a cada 11 anos têm mostrado aumento na proporção de gestantes que inicia o pré-natal cada vez mais precocemente, realizam seis ou mais consultas e 
são submetidas a um número cada vez maior de exames clínicos e laboratoriais básicos 11 .

Nas áreas estudadas, houve substancial melhora do pré-natal oferecido tanto em termos quantitativos quanto qualitativos. No entanto, as melhorias alcançadas mostram-se ainda muito distantes de uma assistência pré-natal minimamente adequada, o que já foi conseguido em diversas outras localidades brasileiras 11,12,13,14. Por exemplo, em Criciúma 12, Estado de Santa Catarina, em 1996, e em Pelotas 11, em 2004, praticamente todas as mães iniciavam o pré-natal e pelo menos três quartos delas realizavam seis ou mais consultas pré-natais, enquanto nas áreas aqui estudadas, isto ocorria em 2005 , para somente $90 \%$ e $52 \%$ delas, respectivamente.

O fato de realizar pré-natal e, principalmente, iniciar as consultas no primeiro trimestre é extremamente importante para detectar precocemente gestações consideradas de alto risco. O início precoce do acompanhamento pré-natal permite intervir sobre causas como, por exemplo, retardo de crescimento intra-uterino, parto prematuro e morte da criança e da mãe, eventos não raros na gestação, pelo menos nos países em desenvolvimento 15 .

O número médio de consulta pré-natal nas áreas estudadas passou de 4,7 em 2002 para 5,5 em 2005. Esta melhora em tão pouco tempo pode ser atribuída a um conjunto de fatores, dentre eles o aumento da renda familiar, maior nível de escolaridade das mães e maior oferta de serviços de saúde, sobretudo pelo aumento da cobertura do Programa Saúde da Família e pelo PHPN, presente, nos últimos anos, na grande maioria dos municípios brasileiros 5,11.

Em relação à realização de exames laboratoriais, que indicam a qualidade do pré-natal recebido, a diferença entre os resultados obtidos nestas áreas e os observados em outras localidades brasileiras é maior ainda. Enquanto em 2002 nove de cada dez mães em Caxias do Sul, Estado do Rio Grande do Sul 13; e Juiz de Fora, Estado de Minas Gerais 14, realizavam exame para detecção de sífilis e anti-HIV, somente $15 \%$ das mães aqui estudadas o fizeram em 2005. Estas mesmas diferenças foram observadas em relação à realização de exame ginecológico, exame das mamas e orientação quanto à amamentação. Os percentuais observados para Criciúma 12, em 1996 e em Pelotas 11, em 2004 são muito superiores àqueles observados para estas mães em 2005.

Um indicador de qualidade do pré-natal é a imunização completa contra o tétano neonatal, que também apresentou melhora substancial no período. Apesar disso, $85 \%$ dos casos de tétano neonatal, em 2004, em todo o país, ocorreram nas regiões Norte e Nordeste ${ }^{4}$. Por esta razão, esforços devem ser intensificados no sentido de aumentar ainda mais esta cobertura.

Houve aumento substancial na realização de exames que dependem de equipamentos, tais como esfigmomanômetro, balança e sonar Doppler. Já para procedimento que dependem mais da habilidade do médico ou da enfermeira, como, por exemplo, o exame ginecológico, exame de mamas ou orientar sobre amamentação, houve declínio importante. Isto sugere que atividades que demandam maior habilidade do profissional, bem como maior tempo de consulta, deixam, muitas vezes, de ser realizadas.

A realização de exames para detecção de doenças sexualmente transmissíveis também aumentou significativamente, mas suas coberturas ainda estão muito aquém do desejado. Isto é bastante grave visto que se o tratamento contra a sífilis for instituído precocemente, esta doença poderá ser curada e menores serão as chances de a criança ser portadora de sífilis congênita. Situação similar ocorre em relação à infecção por HIV que, se adequadamente monitorada durante gestação, menor será a probabilidade de transmissão do vírus à criança. Neste estudo observou-se que, mesmo ocorrendo um crescimento importante na realização destes exames, os índices observados são ainda muito baixos.

O aumento expressivo da realização de ultrasom na gestação está seguindo a tendência de outras regiões, como demonstrado no estudo de coorte na cidade de Pelotas, onde praticamente todas as mães de 2004 realizaram o exame 16 . Outros estudos também demonstram que o ultra-som tem sido realizado mais freqüentemente do que outros exames mais simples e com maior impacto na saúde da criança 13,14 . A predileção por este tipo de exame, apesar da sua pouca utilidade na maioria das situações, é algo que precisa ser mais bem estudado, tanto do ponto de vista do médico quanto das usuárias. Estudos qualitativos teriam muito a contribuir neste sentido.

Chama atenção ainda o fato de um quinto das crianças terem nascido fora do ambiente hospitalar, o que implica ausência de cuidado médico adequado. Isto aumenta sobremaneira o risco de complicação e morte, tanto para o bebê quanto para a gestante. A maioria dos municípios estudados não possuía maternidade ou hospital. Não bastasse a distância às vezes grande até a sede do município, havia ainda, em alguns casos, a necessidade de deslocamento até um outro município. Somam-se a isso a grande dificuldade de transporte público nestas áreas, a impossibilidade de a família pagar por este serviço e a crença de que nascer em casa é o esperado.

A Organização Mundial da Saúde (OMS) recomenda que não mais que $15 \%$ dos nascimen- 
tos ocorram por cesariana. No estudo da coorte de Pelotas em 2004, a taxa de cesarianas foi de $45 \%$ 11. Nos resultados aqui apresentados, a ocorrência de cesariana passou de 13\% em 2002 para $16 \%$ em 2005 , ou seja, está muito próximo ao preconizado pela OMS. Resta saber se estas cesarianas ocorreram entre aquelas de maior risco ou, como observado em vários outros estudos, foram realizadas entre as mais ricas, em geral de menor risco 11. Análise bivariada para 2002 mostrou que a taxa de cesariana entre mães com renda familiar inferior a um salário mínimo foi de $12 \%$ contra $17 \%$ entre aquelas com renda superior a dois salários mínimos ( $p=0,287$ ); em 2005, esta diferença foi ainda maior, passando de $13 \%$ entre as de menor renda para $19 \%$ entre as de maior renda $(p=0,009)$. Consideran- do que as mães de menor renda apresentam os maiores riscos, é possível que as cesarianas não sejam realizadas entre as mães que mais se beneficiaram deste procedimento, o que é muito semelhante ao observado em localidades mais desenvolvidas 1,11 .

Em síntese, este estudo mostrou que as famílias estudadas aumentaram seu poder de compra e melhoraram suas condições de moradia. O prénatal por elas realizado melhorou bastante em termos quantitativos, mas pouco em termo qualitativos. Evidencia-se, portanto, a necessidade de manter estas conquistas e de envidar esforços no sentido de aumentar o acesso aos serviços de saúde e, conseqüentemente, melhorar a qualidade dos cuidados oferecidos.

\section{Resumo}

Em 2002 e 2005 realizou-se inquérito transversal em áreas pobres das regiões Norte e Nordeste do Brasil visando avaliar a assistência à gestação e ao parto entre mães de menores de cinco anos. Foram estudadas 1.528 e 1.529 crianças em 2002 e 2005, respectivamente. Neste período, a escolaridade média das mães aumentou em 1,5 anos, a renda familiar em $R \$ 100,00 e$ o fornecimento de água tratada passou de $23 \%$ para 41\%; a realização de seis ou mais consultas de pré-natal aumentou de $42 \%$ para $52 \%$ e a imunização contra tétano neonatal de 54\% para 77\%; a avaliação da altura uterina de $54 \%$ para $77 \%$ enquanto a realização de teste para HIV e sífilis duplicou, passando de cerca de $8 \%$ para aproximadamente $16 \%$ e de ultra-sonografia de $44 \%$ para $64 \%$. No entanto, a realização de exames ginecológicos caiu de $41 \%$ para $31 \%$ e as orientações sobre amamentação de $66 \%$ para $55 \%$. Apesar de melhorias expressivas na maioria dos indicadores estudados, verifica-se que ainda estão abaixo das regiões mais desenvolvidas do país. Esforços devem ser feitos visando reduzir estes diferenciais.

Assistência à Saúde; Gravidez; Parto; Saúde MaternoInfantil

\section{Colaboradores}

M. A. D. Chrestani analisou dados e redigiu o artigo. I. S. Santos auxiliou na análise de dados e redação de artigo. J. A. Cesar definiu delineamento, coordenou o trabalho de campo, auxiliou na análise de dados e na redação do artigo. L. S. Winckler atuou na revisão e digitação dos questionários e auxiliou na redação do artigo. T. S. Gonçalves supervisionou a coleta de dados e a redação final do artigo. N. A. Neumann auxiliou na definição do delineamento, na análise de dados e na redação final do artigo.

\section{Agradecimentos}

Estudo financiado pela Pastoral da Criança, Fundo das Nações Unidas para a Infância e Adolescência e Programa Criança Esperança (Rede Globo de Televisão/Organização das Nações Unidas para a Educação, a Ciência e a Cultura). 


\section{Referências}

1. Victora CG. Intervenções para reduzir a mortalidade infantil pré-escolar e materna no Brasil. Rev Bras Epidemiol 2001; 4:3-69.

2. Fundo das Nações Unidas para a Infância. Situação mundial da infância 2006. Brasília: Fundo das Nações Unidas para a Infância; 2006.

3. Portal de Serviços e Informações do Governo. Sistema de Informações de Mortalidade. http://www. e.gov.br/defaultCab.asp?idservinfo=3912\&url=http: //www.datasus.gov.br/catalogo/sim.htm (acessado em 17/Mar/2007).

4. Portal de Serviços e Informações do Governo. Sistema de Informações de Nascidos Vivos. http:// www.e.gov.br/defaultCab.asp?idservinfo=3911\&ur l=http://www.datasus.gov.br/catalogo/sinasc.htm (acessado em 17/Mar/2007).

5. Ministério da Saúde Projeto de avaliação nacional do Programa de Humanização do Pré-natal e Nascimento (PHPN): relatório final 2004. Brasília: Ministério da Saúde; 2004.

6. Victora CG, Vaughan JP, Barros FC, Silva AC, Tomasi E. Explaining trends in inequities: evidence from Brazilian child health studies. Lancet 1999; 356:1093-8.

7. Silva IS. Cross-sectional surveys. In: Silva IS, editor. Cancer epidemiology: principles and methods. Lyon: International Agency for Research on Cancer/World Health Organization; 1999. p. 213-29.

8. Victora CG, Barros FC, Cesar JA, Horta BL, Lima SM. A Pastoral da Criança e a saúde materno-infantil em dois municípios do Maranhão 2001. Brasília: Fundo das Nações Unidas para a Infância; 2001.
9. Neumann NA, Victora CG, Halpern R, Guimarães PRV, Cesar JA. Desempenho da Pastoral da Criança na promoção de ações de sobrevivência infantil e na educação em saúde em Criciúma, uma cidade do sul do Brasil. Rev Panam Salud Pública 1999; 5:400-10.

10. Instituto Brasileiro de Geografia e Estatística. Pesquisa Nacional por Amostra de Domicílios, 2005. http://www.ibge.gov.br/home/estatistica/ populacao/trabalhorendimento/pnad2005/ tabbrasil.shtm?c=3 (acessado em 17/Mar/2007).

11. Cesar JA, Matijasevich AM, Santos IS, Barros AJD, Costa JSD, Barros FC, et al. Utilização de serviços de saúde materno-infantis em três coortes de base populacional no Sul do Brasil, 1982-2004. Cad Saúde Publica; no prelo.

12. Neumann NA, Tanaka OU, Victora CG, Cesar JA. Qualidade e equidade da atenção ao pré-natal e ao parto em Criciúma, Santa Catarina, Sul do Brasil. Rev Bras Epidemiol 2003; 6:307-18.

13. Trevisan MR, De Lorenzi DRS, Araújo NM, Ésber K. Pefil da assistência pré-natal entre usuárias do Sistema Único de Saúde em Caxias do Sul. Rev Bras Ginecol Obstet 2002; 24:293-9.

14. Coutinho T, Teixeira MTB, Dain S, Sayd JD, Coutinho LM. Adequação do processo de assistência pré-natal entre usuárias do Sistema Único de Saúde em Juiz de Fora, MG. Rev Bras Ginecol Obstet 2003; 25:71724.

15. Victora C, Barros FC, Vaughan JP. Epidemiologia da desigualdade. São Paulo: Editora Hucitec; 1988.

16. Barros FC, Victora CG, Barros AJ, Santos IS, Albernaz E, Matijasevich A, et al. The challenge of reducing neonatal mortality in middle-income countries: findings from three Brazilian birth cohorts in 1982, 1993 and 2004. Lancet 2005; 365:84754.

Recebido em 19/Mar/2007

Versão final reapresentada em 27/Jul/2007 Aprovado em 13/Nov/2007 